\title{
Stimulating Students to Learn with Accuracy Counter based on Competitive Learning
}

\author{
Ko-Kang Chu, Maiga Chang', Yen-Teh Hsia \\ kirk@ms2.hinet.net,maiga@ms2.hinet.net,hsia@ice.cycu.edu.tw \\ Dept. of Electronic Engineering, Chung-Yuan Christian Univ. \\ ${ }^{1}$ Program Office of National Science and Technology Program for eLearning in Taiwan
}

\begin{abstract}
In this paper we use an Accuracy Counter (AC) to develop the "Web Design" course and to be the evaluation way for measuring and recording the learning status of students. The counts recorded by AC are used to be one of the grading criteria for the students' performance. Students can look others' achievement via the AC website, and may want to compete with others either they are classmates or not. Through the counts recorded by $A C$ and the final scores given from other learners, this paper evaluates the possible competitive conditions and its relations between counts and scores.
\end{abstract}

\section{Introduction}

This paper tries to verify and visualize the learning effects and competitive behaviors in the learning process. In order to achieve our goal an Accuracy Counter (AC) is necessary. Section 2 describes the reasons that the Accuracy Counter is secured for being the criteria of scoring students' achievement in designing a website. The "Web Design" course is used for verifying our assumption and designed in Section 3. There several interesting findings are discovered by Section 4 . Section 5 makes a simple conclusions and names several possible future research issues.

\section{Accuracy Counter}

In general, users will not tend to repeatedly access the same webpage within a short period. Therefore, when the same computer accesses the same webpage again in a short time, it is feasible to consider this connection attempt as "unreasonable". The AC system is designed to block increments of the counter's value under such circumstances. Detail analysis of AC could be found in [1].

Figure 1 demonstrates the architecture of the Accuracy Counter. As we can see in Figure 1, the AC system is a three-tier secured counter. Although there are several modules or functionality, there are four major stages to ensure the data stored in the $\mathrm{AC}$ is correct. These four major stages compose the operation flows of the AC.

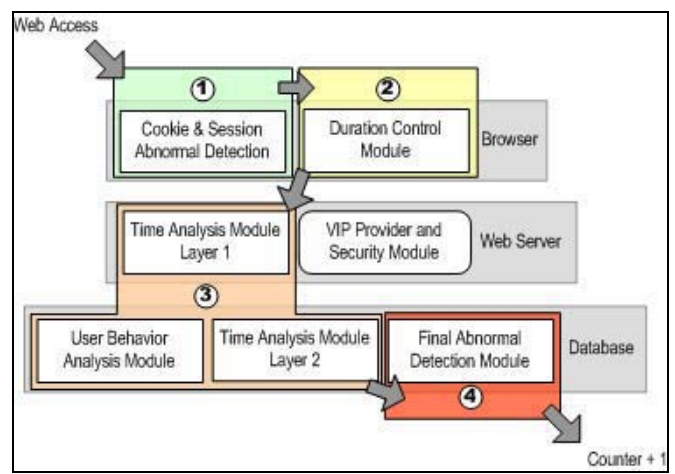

Figure 1. Three-tier architecture of the AC

The concrete operation flows can be draw as Figure 2 shown below. To better understand the survival rate and performance of the AC system, we established a conflicting environment to test it. The system was tested for about four months. In addition to an $\mathrm{AC}$, we also put a traditional counter (TC for short) on each webpage. However, the TC was invisible. We set $\delta$ to be 15 minutes and time-span threshold to be 10 seconds. At the end of the test, the blocking rate of $\mathrm{AC}$ was $61 \%{ }^{1}$

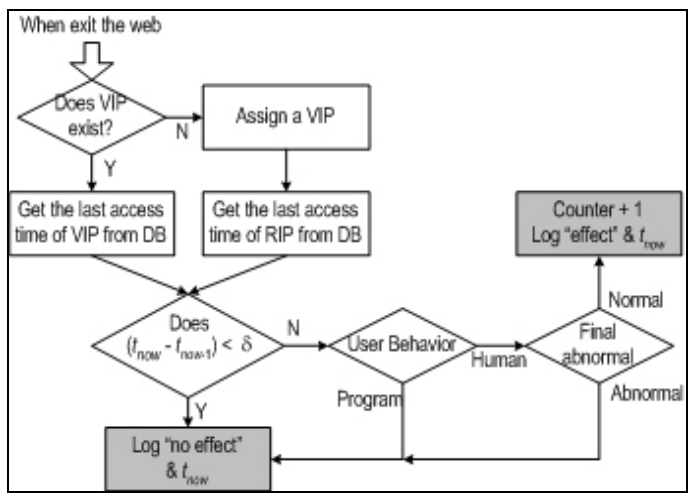

Figure 2. The operation flow of the AC system.

\section{Curriculum Design}

The course we used for verifying the effects of

1 The formula is $(G C-A C) / G C \times 100 \%$ 
competitive learning is "Web Design" in Chung-Yuan Christian University. There were four such classes and forty teams altogether. In addition to exams, the web-designed course usually provides an assignment which asks students to design web sites. We believe it will be helpful for students. However, we encounter two problems: how to stimulate students' interests on this assignment and how to grade it objectively?

In order to solve those problems above, we redesigned the homework in the "Web Design" course. We put a web counter into each group's website. This counter recorded two values, one is the pageviews, and the other is the average visit time on the whole website. To prevent someone from increasing the counter's value by using devious ways, we used the Accuracy Counter to record these two values.

We assume that students would care the pageviews, the average duration time and his/her place in the class. Besides, we told the students, the pageviews and average duration time will be part of their final score. In other words, we use AC to increase the motivation.

In order to attract more people to visit their websites, every student must promote their web site hardly. But when they begin to advertise, they have to enrich the contents first. That because if the contents of a website are pool, we can then imagine visitors will leave the website quickly, so the average duration time will be down. This means of the "GOOD" website is the most people who love it. Finally we use the pageviews and average duration time to evaluate these websites.

However, we cannot use accuracy counter to fully replace the adjudicator. We know some students are used to procrastinate or not good at how to advertise, so the pageviews may be lower but the quality of the website is better. Therefore, we did not give up evaluating the web by a teacher. But we made a little change. We let different groups from different classes to evaluate others works.

\section{Experiment Results and Discussions}

In our experiment, there are FOUR classes from TWO universities (CYCU and ShengTe). The four classes are Dept of Special Education (from CYCU), Dept. of International Trade (2 classes from CYCU) and Dept. of Mass Communication (from ShengTe). The grading criteria divided into two parts: data stored in $\mathrm{AC}$ and average score gave from other three groups (selected from different classes and schools randomly.)

Before we build the relations between the competitive learning and the learning achievement (final score) of students, we draw down the trends of pageviews as Figure 3 to Figure 5 illustrated below. Each figure in Figure 3 to Figure 5 represents one kind of trends. As we can see the $\mathrm{X}$-axis in Figures (3-5) indicates week number. The semester in Taiwan usually takes 18 weeks. After bypass the first several weeks while still teaching students fundamental skills and two major exams (midterm and final exam), we left only about 13 weeks to let students establish and promote their websites. The Y-axis respectively indicates the counts that their website gets in the specific week. The descriptions shown on the above in every figures likes sea04(4674, 113, 24/36, 6/11) means GroupID (pageviews, average duration time, place in all groups, place of in its class).

Therefore, based on the data stored in $\mathrm{AC}$ we find there are three kinds of students. One kind of them are quite aggressive - Early Activation; One is meticulous in their works - Later Activation; One is most of groups being (no rush and no worry) - Normal Activation.

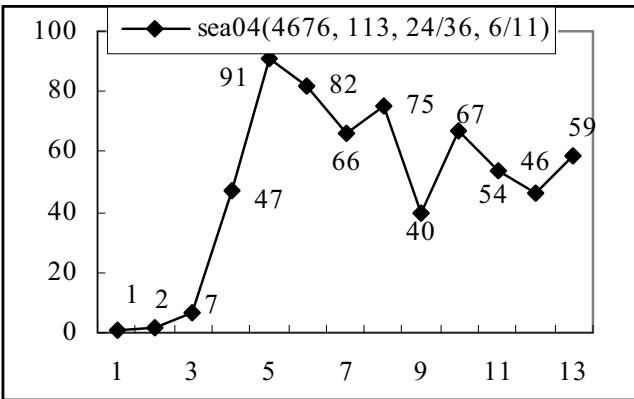

Figure 3. Trend Category I : Early Activation

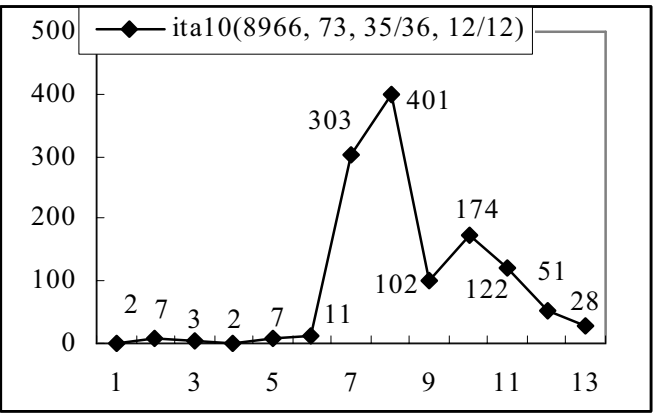

Figure 4. Trend Category II : Normal Activation

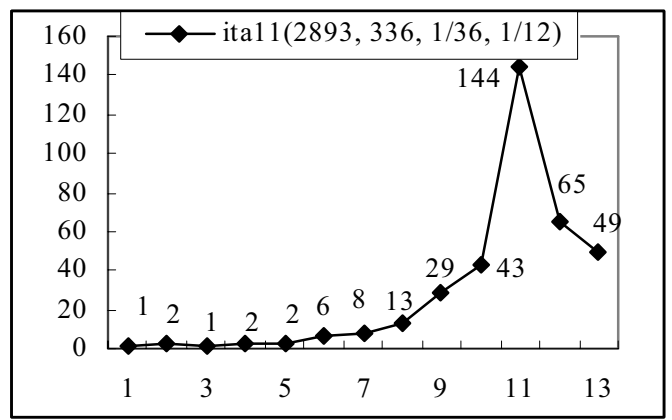

Figure 5. Trend Category III : Later Activation

By using these three trends categories, the correlation analysis could be applied to all groups as Table 1 lists below. The correlation is high if two AC trends from different groups are positive relative, on the other hand, the correlation will be low and even be negative if two AC trends are no relation or irrelevant. Herein we discover a very interesting finding, that is the score is either very 
high (leading place) or very low (straggling place) if the average correlation coefficients (corrcoef) is low as Figure 6 shown below.

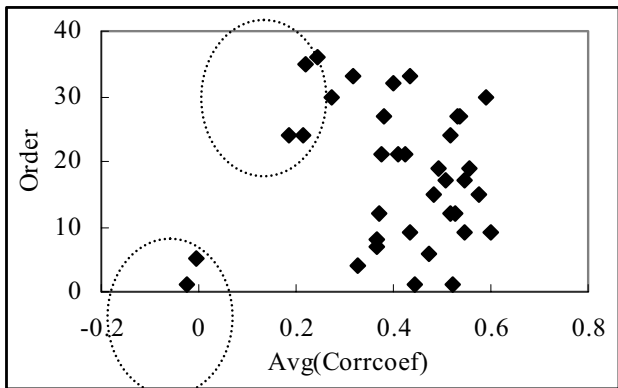

Figure 6. Relations between Place and Corrcoef

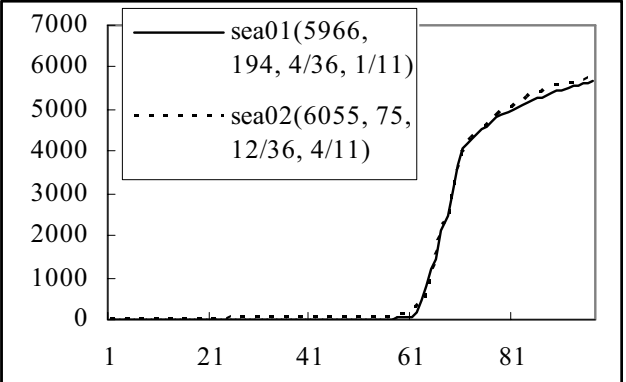

Figure 7. The classical Competitive Behaviors between two groups

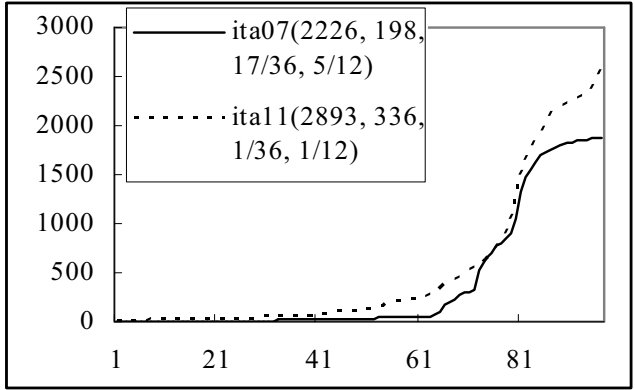

Figure 8. The give up example

The second finding is the competitive behaviors occur between two groups. Figure 7 represents below is a classical example. Since they are competing, their final score is near. The third finding is also trivial but you can not easily prove before applying the Information
Technology mechanism. As Figure 8 shown below, we can find out the group ita07 compete with the group ita11 in first phrase. However, since the group ita11 did a very good job, the differences between ita07 and ita11 is more and more large. Finally, as we can see the group ita07 gives up.

\section{Conclusions}

In this paper we try to apply an accuracy counter (AC) to verify the effect of competitive learning. After using the correlation analysis methods, there are four findings discovered:

a. The score is either very high (leading place) or very low (straggling place) if the average correlation coefficients (corrcoef) is low.

b. The continuously competitive behaviors will let both of groups get higher scores.

c. If the differences between two competitive groups become large, the lower achievement group will give up.

d. Two groups from different classes competes with each other will make similar achievement at final.

There are three possible directions for researching:

a. How can we positively sure the competitive behaviors are occurring between the two groups that we said? (subscribing competitor's info)

b. Is it possible to force students to compete by using AC or other IT mechanism? (competitive/challenge letter)

c. The competitive behaviors should be a time-sequence issue, that is, once group A can not defeated then change our target to group B. (time-slide window)

\section{References}

[1] Ko-Kang Chu, Maiga Chang and Yen-The Hsia, "Designing a Mechanism to Assure the Reasonableness and Fairness of Information Stored in Databases on WWW," IEEE International Conference on Systems, Man and Cybernetic 2003, (IEEE CSMC 2003), Washington, D.C., USA, October 5-8, 2003, pp. 2302-2307

Table 1. Correlation Table for different gourps

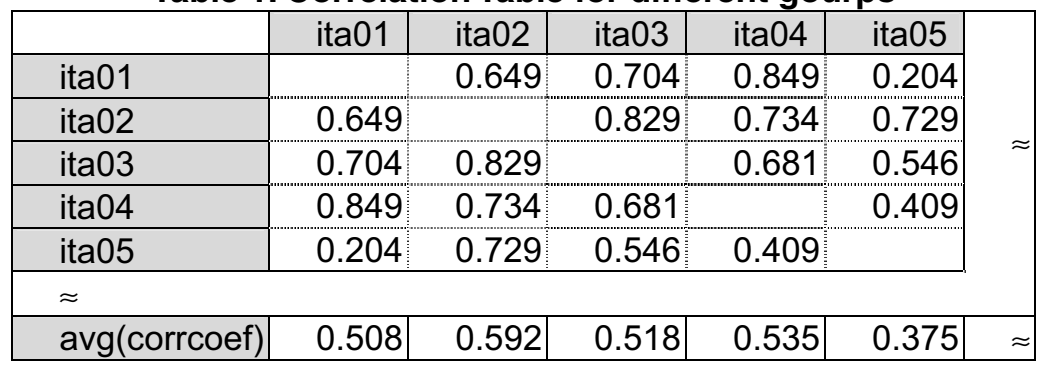

